\title{
PROPERTIES OF OBTURATIONS AND OBTURATION MATERIALS, ASSOCIATED WITH THE DEVELOPMENT OF SECONDARY CARIES
}

\author{
Silviya Dimitrova, Nadia Bibova \\ Department of Operative Dentistry and Endodontics, Faculty of Dental Medicine, \\ Medical University - Plovdiv
}

\begin{abstract}
The main part of dentists' work is associated with the operative treatment of dental caries, aiming at the stabilization of the process and restoration of the natural anatomy and function of the teeth with artificial materials. With the emergence of restorative dental medicine came the questions related to the survival rate of the restorations, the reasons for their replacement and the possibilities for control and management of the factors, leading to activation and progression of the carious process.

The aim of the literature overview is to study the most common reasons, leading to a decrease of the survival rate of different restorations within the conditions of the mouth cavity. It analyzes factors with direct and indirect importance for the development of secondary caries and the longevity of obturations, such as polymerization shrinkage, accumulation of dental biofilm, micropermeability, marginal adaptation, microgap, etc.
\end{abstract}

Keywords: secondary caries, marginal adaptation, micropermeability, biofilm

The replacement of restorations is the most common operative approach, used for both stabilization of the carious process, and prevention of its progress (1). When the operative treatment is accepted without control of the etiological factors, new caries lesions may be developed around the restorations, leading to re-restoration of the tooth (1). In the presence of different marginal defects about three quarters of all restorations are replaced in order to prevent further damage (2). The reasons for operative treatment are of great interest, and demonstrate the ne-

\footnotetext{
Address for correspondence:

Silviya Dimitrova

Faculty of Dental Medicine

Medical University - Plovdiv

3 Hristo Botev Blvd

4000 Plovdiv

e-mail:silvi_dok@yahoo.com
}

Received: March 23, 2017

Accepted: June 27, 2017 cessity of reevaluation of the expenses for operative treatment of dental caries, which accumulate during the lifetime of the patient, compared to the expenses for individual preventive non-operative treatment (3).

Regarding the occurrence and the development of caries adjacent to obturation or sealant, in the literature there are discussions of different factors related to the obturation materials and the restorations (4). Every obturation has limited time of functional suitability and could be compromised by different objective factors, associated with the obturation materials, the condition of the adjacent hard tissues, affected surfaces, the skills and the equipment of the dentist, general and/or oral health of the patient, etc. (5).

\section{Marginal Adaptation, Microgap}

It has been discussed for a long time that marginal gaps lead to the development of secondary caries, and that the marginal adaptation of the restorative material to the cavity wall is very important (6). It is suggested that if there is a marginal gap, the dentin- 
al tubules are not closed, the bacteria can easily penetrate, the dissemination of secondary caries is higher, and the survival of the restorations is short. The most common location of caries along the obturation margins is the gingival edge in class II to class $\mathrm{V}$ obturations, where plaque is accumulated, as well as at the border obturation - cavity wall. The occlusal marginal edges, even overcontoured and with plaque retention, rarely develop caries, probably because of the better capacity for plaque control $(7,8)$. The main factors affecting the occurence of secondary caries along the gingival margin of the obturations are: the restorative technique, the quality of the obturation material and the plaque control by the patient (9), as it largely depends on the preparatory technique $(5,10)$. The gingival marginal edge of the cavity is considered to be susceptible to contamination from infiltration of gingival fluid and saliva between the obturation and the cavity wall, especially if it has subgingival location and no rubber dam is used for isolation. The presence of defects resulting from the adaptation of obturation creates preconditions for subsequent retention and accumulation of dental plaque, and respectively for appearance and development of secondary caries $(3,7,11)$. The larger the subgingival depth (2), and the wider the cavity, the smaller the possibility to guarantee precise marginal adaptation is, and the more often generalized attrition, fractures and microgaps occur $(10,12)$. Another significant moment is the lack or the underestimation of the moisture control. It is known that contamination with fluids has much more serious consequences for the composite than for the amalgam obturations. A number of researchers report that no more than $15 \%$ of the dentists use rubber dam and without it the clinical protocol, focusing on dry operative field, cannot be followed successfully $(5,12)$. Marinova reports that, during their in vitro study, none of the materials demonstrated complete lack of microgaps and micropermeability, which is more expressed at the borders of the dentin (13). Goldberg et al. report increased probability for secondary caries with deterioration of the quality of the margin on smooth surfaces, but also that oral hygiene is an important factor for the risk of secondary caries (14). According to Hamilton et al. (15), however, the margin deterioration is not a predictor of the lifespan of the restoration. In a later literature review, Goldberg (16) speaks of the complexity of finding a clear connection between the marginal gaps and secondary caries, and Söderholm et al. (17) do not find significant difference in the size of the marginal distance on areas without secondary caries, compared to areas with secondary caries in amalgam restorations. Kidd and Beighton report that only gaps over $0.4 \mathrm{~nm}$ with composite obturations lead to an increased amount of microorganisms in the dentin. Compared to areas with secondary caries, the areas with large gaps have similar levels of microorganisms, but lower levels of Streptococcus mutans (18). Gaengler et al. (19) come to the conclusion that the imperfections in the integrity of the margin of composite restorations do not increase the risk of secondary caries, however, Hayashi et al. report that the deterioration of the margin and the marginal coloration in composite restorations is associated with more frequent future damages (20). The presence of a microgap between the obturation and the cavity wall is considered to be a positive indication for the development of a wall lesion. This subject is still discussible, as some studies find positive correlation between the size of the gap and the appearance of secondary caries $(21,22)$, while others do not find such relation (8). The microgaps at the margin obturation-tooth, which are smaller than 35-50 $\mu \mathrm{m}$, do not predispose to development of secondary caries, unlike the larger ones (8). The marginal gaps allow penetration of microorganisms and accumulation of plaque, which will lead to further demineralization of the cavity wall (21). In an in-vitro study Nassar and González-Cabezas (2011) study the significance of the gap size and its geometry as a risk factor for the development of secondary carious lesions, particularly wall lesions (23). Their results completely confirm the above-mentioned and demonstrate that the presence of an additional gap at the dentinal wall area does not affect secondary caries development, as long as the enamel gap is smaller or about $30 \mu \mathrm{m}$. According to the authors, the probable cause is the larger distance of the obturation in the inner third of the dentin, which impairs the exchange and transfer of substrates for the microorganisms, due to the larger distance, which they must pass. On the other hand, due to the delayed exchange, the extracted calcium ions during the demineralization are held longer in the microgap, thus helping the natural process of re-mineralization (23). Therefore, we may conclude that secondary caries is not developed as a result of micropermeability around the ob- 
turation, but is a lesion, similar to primary caries on smooth surfaces (9). The presence of overhanging obturation margins, even small and difficult for clinical detection, predispose to the appearance of secondary caries, which shows that accumulation of dental plaque is an important factor in the etiology of caries adjacent to obturation or sealant. The results of recent studies show that the development of external lesion is probably more significant than the development of wall lesion (9). In their study Mjör and Qvist (1997) have established that combined wall-adjacent and residual lesions are found more often if the preoperative diagnostics finds external lesion in the obturation margin. At the same time, wall lesions, which are found after the removal of the obturation, are not so frequent when there is a marginal defect without caries. These results confirm that the external lesions are more indicative of presence of secondary caries, although the use of external lesion as indication of wall lesion is limited. For example, Grossman and Matejka come to the conclusion that the presence of an outer lesion is a sure indicator for the presence of a wall lesion. The absence of an outer lesion does not mean the absence of a wall lesion; as well as the outer lesion is not an indicator for the size of the wall lesion (24). Perhaps the best proof that the micropermeability and the microgaps do not necessarily lead to secondary caries is the study of enamel cracks. Caries is almost never observed in these cracks or in their adjacent areas, although they also change color, similarly to the margins of esthetic restorations. The coloration is due to the proteins in the cracks, similar to those from the pellicle, which normally covers the teeth and the obturations (11). These results suggest that it is disputable whether there is correlation between the width of the microgap and the development of secondary caries $(14,15,16)$. The quality of the margins does not necessarily increase the risk of secondary caries, although their poor quality, in fact, can reduce the clinical duration of the restorations, due to incorrect diagnosis (2).

\section{Micropermeability, Nanopermeability}

Micropermeability is considered to be one of the main factors for the occurrence of secondary caries, associated with the forming of a microgap (25). A condition for the occurrence of micropermeability is the rupture of the adhesive bond by the forces of polymerization shrinkage of the composite materials, or occlusal load, and to a lesser extent is associated with the impact of the chemical and thermal factors $(26,27,28)$. In theory, microfiltration is possible between all marginal surfaces in the adhesively bonded obturations: dentinal tubule - plastic process - hybrid layer - adhesive layer - composition material. The question regarding which complex adhesive area is most unstable, is debatable. Nakabayashi (4), Pashley et al. (29) suggest that the collagen fibers, completely proliferated, with plastic at the base of the hybrid layer, are an initial location for disintegration. Sano et al. (30) study the micropermeability at the level of hybrid layer, and they did not establish a gap between the marginal layers. The disintegration between the hybrid and the adhesive layer is found to be the weak point in the multi-component bonding surface between the hard dental tissues and the composite materials (31). There are suggestions that the water absorption by the methacrylic polymers of the primer and the hygroscopic expansion could compensate the initial gap. This expansion of the methacrylic polymers, however, is not sufficient to restore the reduction volume of the obturations, due to the polymerization shrinkage (26). Once the gap appears, it is enlarged by the effect of the mechanical factors, stress zones, and the chemical and thermal factors contribute further to the expansion of its size $(26,27,28)$. The physical existence of the gap, clinically and ultramicroscopically determinable, is not pathognomically sufficient criterion for diagnosis of micropermeability (31). The formation of an inadequate hybrid layer, susceptible to degradation, is a result of diffuse-dependent hydrolytic processes, and is called nanopermeability. The presence of nanopermeability is associated with a gap, whose enlargement in most of the cases is combined with reduction of the strength of the bond (32). It is proven that the factor with greatest importance for opposition against the forces of the polymerization shrinkage, is the strength of the adhesive bond $(26,27)$, which is still problematic (33).

\section{Polymerization Shrinkage}

The polymerization shrinkage $(1.5-3 \%$ according to McCabe, 2008) is suggested to cause secondary caries, due to defects of the margins, which appear during contraction of the composite mate- 
Silviya Dimitrova, Nadia Bibova

rials $(5,34)$. The presumption, that the shrinkage of the polymer causes secondary caries is based on a simplified model, according to which the contraction causes loss of adhesion and leads to gaps in the margins, allowing the penetration of bacteria $(5,25)$. There is no solid evidence that the shrinkage of the polymer has direct or indirect effect on the etiology of the secondary caries, and it is arguable if its elimination will have a significant effect on reducing the risk of the secondary carious process (2). The colored marginal distances may increase the possibility of incorrect diagnosis and lead to unnecessary change of restorations, thus the polymerization shrinkage may have indirect negative effect on the clinical durability of the composite restorations $(2,12,34)$. The polymerization shrinkage is also related to the C-factor (configuration factor), formed on the basis of the ratio between the bound and unbound surfaces of the cavity. It is assumed, that the incremental application of the composites helps overcome the residual polymerization stress, proportionally related to the C-factor $(5,26)$. The polymerization shrinkage may result in appearance of cracks and retraction of the material from the gingival margin, due to the weaker adhesion of the bonding agents to the dentin and the cement, than to the enamel and with occlusal direction of the light source $(5,12)$. These gingival parts of the obturation are also more difficult to clean by the patient, compared to the other tooth surfaces, especially with approximal location (11). Unlike the obturations with dental amalgam, the composite obturations allow no mistakes, and despite the intensive studies, the problem with the marginal gaps, and respectively the secondary caries of composite restorations, is still unsolved (13). At the same time, other authors report that the initial formation of plaque on hard surfaces is mostly affected by the environment in the oral cavity, than by the parameters of the obturation materials.

\section{Accumulation of Plaque Biofilm}

Outer lesions with secondary caries are proven to be associated with the presence of plaque biofilm on the margins of the restorations, which depends on the type of the obturation material $(8,22)$. Microbiological studies of secondary carious lesions do not establish significant differences in the microbial flora between primary and secondary caries (18). Accord- ing to different studies, composite materials accumulate more and more carious plaque, compared to dental amalgam, silicate cements and glass-ionomer cements. It has been proven that the aging composites become favorable environment for microorganisms and contribute to the accumulation of more biofilm than the other obturation materials $(35,36)$, and the acids from the biofilm increase the risk of development of a carious process. Under composite restorations with gaps, there have been found eight times more anaerobic cariogenic microorganism than under defective obturations with dental amalgam with underlay from zinc-oxy-phosphate cement (8). Many studies prove that composites, including composite cements, have markedly weaker bacteriostatic potential than materials containing silver, gold, tin, copper, zinc oxide, etc. Kidd and Beighton (18) studied the connection between the marginal coloration of composite obturations and the microbial levels in the dentin. They established that $79.5 \%$ of the areas with soft carious dentin were under colored margins, and $55.5 \%$ of the areas with hard dentin were also found under colored margins. Except for categorically proven carious lesions, none of the clinical indicators can predict the presence of soft carious dentin under marginal coloration. On the other hand, significant connections have been established between microorganisms from the marginal biofilm, and microorganisms found in the dentin. There are more microorganisms in the marginal plaque over areas with soft carious dentin than areas with hard dentin. These data show that the marginal gaps and the marginal coloration, which are not determined as carious lesions, do not suggest secondary caries, and the microbial levels of the marginal plaque correlate with the presence of carious dentin (2).

\section{REFERENCES}

1. Braga MM, Chiarotti AP, Imparato JC, Mendes FM. Validity and reliability of methods for the detection of secondary caries around amalgam restorations in primary teeth. Braz Oral Res. 2010;24 (l):102-7. doi: 10.1590/S1806-83242010000100017

2. Sarrett DC. Prediction of Clinical Outcomes of a Restoration Based on In Vivo Marginal Quality Evaluation. J Adhes Dent. 2007; 9 (Suppl 1):117-120. 
3. Özer L, Thylstrup A. What is known about caries in relation to restorations as a reason for replacement? A review. Adv Dent Res. 1995; 9 (4):394-402.

4. Nakabayashi N. Dental biomaterials and the healing of dental tissue. Biomaterials. 2003; 24 (13):2437-2439. doi: 10.1016/S0142-9612(03)00112-1

5. Filipov I. Investigation of key problems in the photopolymerization of composite materials in obturation of the second class of cavities. Dissertation. Plovdiv; 2006. (in Bulgarian)

6. Franco EB, Benetti AR, Ishikiriama SK, Santiago SL, Lauris JRP, Jorge MFF, et al. 5-year clinical performance of resin composite versus resin modified glass ionomer restorative material. Oper Dent. 2006; 31(4): 403-408. doi: 10.2341/05-87

7. Peneva M. Tooth decay in the 21st century. Sofia:East-West; 2008: 69-71. (in Bulgarian)

8. Rimalovska S, Kukleva M. Secondary caries in temporary and permanent teeth - literary review - part 1. Dental medicine. 2012; 94(3):204-212. (in Bulgarian)

9. Mjör IA, Toffenetti F. Secondary caries: a literature review with case reports. Quintessence Int. 2000; 31(3):165-179.

10. Yamazaki A, Hibino Y, Honda M, Nagasawa Y, Hasegawa Y, Omatsu J et al. Effect of water on shear strength of glass ionomer cements for lutting. Dent Mater. 2007; 26 (5):708-712. doi: 10.4012/ dmj.26.708

11. Mjör IA. Clinical diagnosis of recurrent caries. J Am Dent Assoc. 2005; 136 (10):1426-33. doi: 10.14219/jada.archive.2005.0057

12. Marinova M. Root caries. Comparative analysis of materials used in its treatment. Dissertation. Sofia; 2012 (in Bulgarian)

13. Marinova M, Karayasheva D, Boteva E. Microgrowth study of the gingival base in the second class of cavities, oblique by means of a technique with different types of aesthetic materials. Dental medicine. 2010; 1:24-28. (in Bulgarian)

14. Goldberg J, Tanzer J, Munster E, Amara J, Thal F, Birkhed D. Cross-sectional clinical evaluation of recurrent enamel canes, restoration of marginal integrity and oral hygiene status. J Am Dent Assoc. 1981; 102(5):635-641. doi: 10.14219/jada. archive.1981.0155

15. Hamilton JC, Moffa JP, Ellison JA, Jenkins WA. Marginal fracture not a predictor of longevity for two dental amalgam alloys: a ten-year study. J Prosthet Dent. 1983; 50(2):200-202.

16. Goldberg AJ. Deterioration of restorative materials and the risk for secondary caries. Adv Dent Res. 1990; 4:14-18. doi: 10.1177/08959374900040010201

17. Söderholm KJ, Antonson DE, Fischischweiger W. Correlation between marginal discrepancies at the amalgam/tooth interface and recurrent caries. In: Anusavice KJ. (ed.) Quality Evaluation of Dental Restorations: Criteria for Placement and Replacement. Chicago: Quintessence; 1989. p. 95-108.

18. Kidd EA, Beighton D. Prediction of Secondary Caries around Tooth-colored Restorations: A Clinical and Microbiological Study. J Dent Res. 1996; 75 (12): 1942-1946. doi: 10.1177/00220345960750120501

19. Gaengler P, Hover I, Montag R, Gaebler P. Micro morphological evaluation of posterior composite restorations - a 10-year report. J Oral Rehabil. 2004; 31(10):991-1000. doi: 10.1111/j.1365-2842.2004.01329.x

20. Hayashi M, Wilson NH, Watts DC. Quality of Marginal Adaptation Evaluation of Posterior Composites in Clinical Trial. J Dent Res. 2003; 82 (1):5963. doi: $10.1177 / 154405910308200113$

21. Cenci MS, Pereira-Cenci T, Cury JA, Ten Cate JM. Relationship between gap size and dentine secondary caries formation assessed in a microcosm biofilm model. Caries Res. 2009; 43(2): 97-102. doi: 10.1159/000209341

22. Totiam P, Gonzalez-Cabezas C, Fontana MR, Zero DT. A new in vitro model to study the relationship of gap size and secondary caries. Caries Res. 2007; 41(6): 467-473. doi: 10.1159/000107934

23. Nassar HM, Gonzalez-Cabezas C. Effect of Gap Geometry on Secondary Caries Wall Lesion Development. Caries Res. 2011; 45(4):346-352. doi: $10.1159 / 000329384$

24. Grossman ES, Matejka JM. Reliability of outer lesion secondary caries as a predictor of wall lesions. Am J Dent. 1999; 12(1): 31-36.

25. Ferracane JL. Developing a more complete understanding of stress produced in dental composites during polymerization. Dent Mater. 2005; 21(1):3642. doi: 10.1016/j.dental.2004.10.004

26. Manchorova N. Postoperative sensitivity in composite obturations 1 and 2 class. Dissertation. Plovdiv; 2009. p. 38-39. (in Bulgarian) 
27. Manchorova N, Vladimirov S. Comparative study of post-operative sensitivity in single and multistep adhesive system obturation. Dental examination. 2007; 89(3):188-194. (in Bulgarian)

28. De Munk J, Van Laduyt K, Peumans M, Poitevin A, Lambrechts P, Braem M, et al. A critical review of the durability of adhesion to tooth tissue: methods and results. J Dent Res. 2005; 84(2):118-32. doi: $10.1177 / 154405910508400204$

29. Pashley DH, Ciucchi B, Sano H, Horner JA. Permeability of dentin adhesive agents.Qint Int. 1993; 24(9):618-31.

30. Sano H, Takatsu T, Ciucchi B, Horner JA, Matthews WG, Pashley DH. Nanoleakage: leakage within the hybrid layer. J Dent Res. 1995; 20(1):18-25.

31. Tay FR, Gwinnet AJ, Pang KM, Wei SH. Variability in microleakage observed in a total-etch wetbonding technique under different handling conditions. J Dent Res. 1995; 74(5):1168-1178. doi: 10.1177/00220345950740050501

32. Okuda M, Pereira PN, Nakajima M, Tagami J, Pashley DH. Long-term durability of resin dentin interface: nano-leakage vs. micro tensile bond strength. Oper Dent. 2002; 27(3):289-96.

33. Stavridakis MM, Dietschi D, Krejci I. Polymerization shrinkage of flowable resin based restorative materials. Operative Dentistry. 2005; 30(1):118-128.

34. McCabe JF, Walls AWG. Resin based filling materials. In: Applied dental materials. Blackwell Publishing Ltd.; 2008. p.195-225.

35. Gonzalez-Cabezas C, Li Y, Gregory RL, Stookey GK. Distribution of three cariogenic bacteria in secondary carious lesions around amalgam restorations. Caries Res. 1999; 33(5):357-365. doi: 16534

36. Svanberg M, Mjor IA, Orstavik D. Mutans streptococci in plaque from margins of amalgam, composite, and glass-ionomer restorations. Dent Res. 1990; 69(3):861-864. doi: 10.1177/00220345900690030601 